\title{
Dynamics of Granular Heaplets: A Phenomenological Model
}

\author{
Yong Kheng Goh ${ }^{1,2}$ and R.L. Jacobs ${ }^{2}$ \\ 1 Department of Mathematical Sciences, University of Essex, \\ Colchester CO4 3SQ, United Kingdom \\ kygoh@essex.ac.uk \\ 2 Department of Mathematics, Imperial College, \\ London SW7 2AZ, United Kingdom \\ r.jacobs@imperial.ac.uk
}

\begin{abstract}
When a discrete granular layer on a uniform substrate is tapped from beneath the material piles up into discrete heaps which gradually coarsen. We investigate the relaxation dynamics of the heaping process. We present a non-linear phenomenological partial differential equation to describe the formation of the heaplets. This equation is derived from the continuity equation for a diffusive powder system and from a constitutive equation giving the current as the sum of three terms: the first proportional to the gradient of the height profile with a limiting factor, the second related to the average curvature of the heap surface and the third related to the Gaussian curvature.
\end{abstract}

\section{Introduction}

When we perturb a layer of granular material a rich variety of interesting phenomena can occur depending on the nature of the perturbation. Examples are subharmonic wave patterns and oscillons in vertically vibrated granular layers 1 2]; compaction and memory effects in tapped tall granular columns [34]; and stratification of a granular mixture flowing down an inclined plane [56]. Even simple experiments such as tapping a thin granular layer, result in interesting phenomena such as the formation of isolated granular "droplets" (heaplets) 7 .

In this article we are particularly interested in a simple system that consists of a thin layer of granular material subjected to a series of discrete taps from beneath. We are interested in constructing a computer model of the dynamics of formation of heaplets in the tapped granular layer. The first section of the article describes an experimental setup in which the phenomena can be observed and also shows typical morphologies that develop. Then we introduce our phenomenological model by constructing a surface free-energy functional. The dynamics of the system is then derived by setting the time-derivative of a density equal to the functional derivative of the free-energy and the result is cast into the form of an equation of continuity. The equation is solved numerically and results are presented. Finally we summarise our results. 

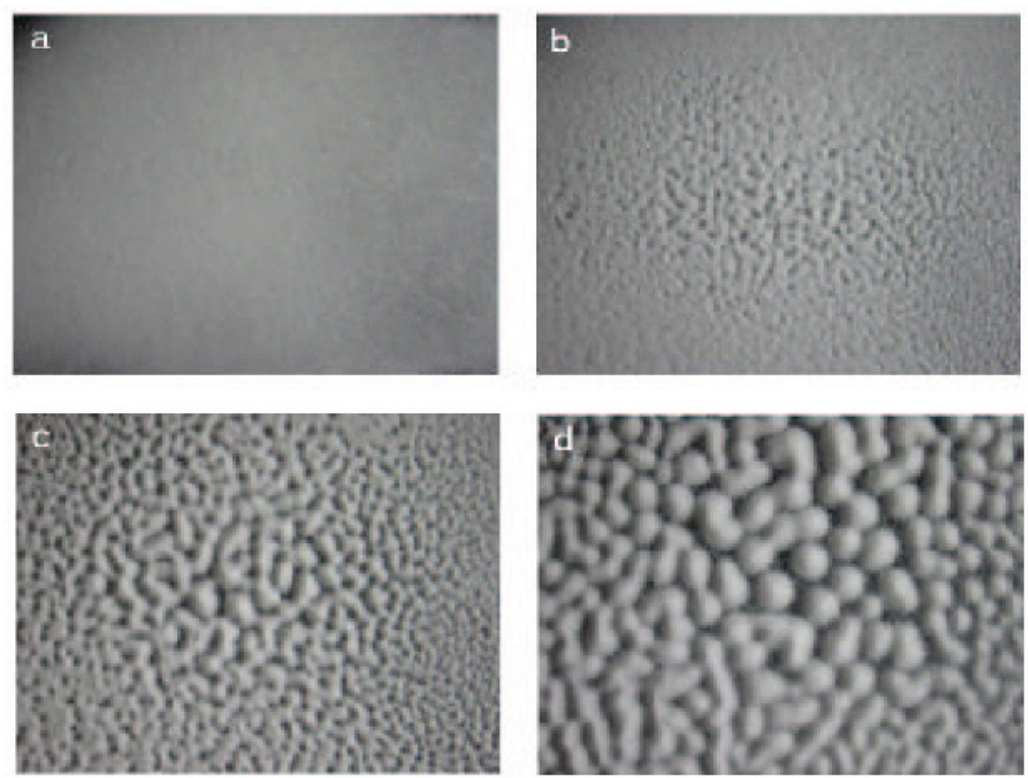

Fig. 1. A layer of silica beads subjected to gentle taps. The first picture (a) is the initial flat layer with pictures (b)-(d) showing the morphology of the layer after $k=20,40$, and 100 taps.

\section{A Simple Experiment}

A homogeneous and thin (1-3 particles depth) layer of silica beads (approximately $30 \mu \mathrm{m}$ in diameter) is prepared on a thin glass plate. The layer is then tapped from below at the center. After each tap we wait for a long enough period until all activity on the surface of the layer has ceased before tapping again. Figure 1 shows a series of photographs obtained from an experiment on a tapped silica layer with nearly constant tapping intensity.

The layer is initially flat as shown in Figure 17. After several taps, the flat layer of silica beads becomes unstable and starts to corrugate (Figure 10). As the number of taps increases, corrugations coarsen and the layer now forms a landscape of ridges (Figure 15). The pattern finally develops into more isolated heaplets (Figure 1 d). The experiment is fairly robust in the sense that the frequency and intensity of the taps do not have to be carefully controlled for the pattern to develop. When the pattern is fully developed, the heaplets become nearly stable against any further taps, with the characteristic size and the rate of the formation of heaplets being proportional to the intensity of the taps.

Duran 8 made a close analogy between the heaplet formation process and the de-wetting process of a layer of water on a glass plate. The value of this analogy can be seen from the above figures where clusters of silica beads slowly coalesce to form small heaplets as if under the influence of surface tension. Of 
course there is no true surface tension since the silica beads interact via hard core repulsions but an effective surface tension can come arise from convective drags [7] or the inelasticity of the particles [9].

\section{Equation of Continuity and Current Equation}

It is useful to have quasi-hydrodynamic equations to describe the dynamics of heaplet formation. However there is no obvious time variable in the problem and we need to take care in the continuum modelling. In the tapped layer there are two distinct phases of activity with different time scales in the dynamics. The first is an excitation phase when the layer is tapped. This is followed by a relaxation phase during which the perturbed layer relaxes towards a meta-stable state. Ordinary clock time cannot be used as a proper time variable for the purpose of continuum modelling, since the actual duration between two consecutive taps is unimportant (provided the system relaxes completely.) What is important is the number of tap cycles that the layer has undergone. Therefore a more useful "time" variable is the number of taps rather than the clock time. In this paper time $t$ refers to a continuum version of the number of taps.

We want to set-up an equation of mass continuity with a constitutive current equation to model heaplet formation on a tapped granular layer. The current equation must describe lateral diffusion of the granular particles to regions of higher density. This leads to an effective attraction between particles and encourages the formation of heaps. However the slope of the side of a static heap cannot exceed a value determined by the angle of repose, $\theta_{\mathrm{r}}$ of the material. This suggests that the effective diffusion is limited by a slope-dependent factor and goes to zero at a critical slope equal to $\tan \theta_{\mathrm{r}}$. Further terms are needed in the current equation and are related to the curvatures of the surface of the layer. These terms should describe the situation when the system is tapped and protrusions are eroded away so that the sides of the heaps become as smooth as possible i. e. the curvature of the surface becomes as small as possible.

Gathering these requirements we introduce a surface free energy functional

$$
\mathcal{F}[\rho]=\int \mathrm{d}^{2} \boldsymbol{x}\left\{-\frac{1}{2} D(\gamma)\left(|\nabla \rho|^{2}-\frac{1}{2} B|\nabla \rho|^{4}\right)+\kappa(\gamma)\left[\operatorname{tr}\left(\rho_{i j}\right)\right]^{2}+\kappa^{\prime}(\gamma)\left[\operatorname{det}\left(\rho_{i j}\right)\right]^{2}\right\}
$$

where the density field $\rho(\boldsymbol{x}, t)$ i. e. the mass per unit area is proportional to the height of the layer assuming no compaction during tapping. $D, \kappa$ and $\kappa^{\prime}$ are positive parameters that depend on the tapping intensity $\gamma$, and $1 / \sqrt{B}=\tan \theta_{\mathrm{r}}$ is the critical slope of the material. Here $\rho_{i j}$ is the Hessian matrix $\rho_{i j}=\partial^{2} \rho / \partial x_{i} \partial x_{j}$. The first two terms mimic the effective attractive and give rise later to a diffusion equation with negative time i. e. one that favours the accumulation rather than the dissipation of heaps. The trace and determinant in the last two terms are the two rotational invariants of the Hessian matrix.

Now we assume that $\rho(\boldsymbol{x}, t)$ evolves so as to minimise the free energy according to model A Langevin dynamics, in Gunton's terminology [10, and this gives 
an equation of motion of form

$$
\frac{\partial \rho}{\partial t}=-\zeta \frac{\delta \mathcal{F}}{\delta \rho(\boldsymbol{x}, t)},
$$

where $\zeta$ is a rate constant that sets the time scale of the growth of the pattern and is set to unity by choice of units. $\mathcal{F}$ is defined as in (1). Taking the functional derivative of the free energy and substituting into (2) we get

$$
\frac{\partial \rho}{\partial t}=-\nabla \cdot\left\{D(\gamma)\left(1-B|\nabla \rho|^{2}\right) \nabla \rho+\kappa(\gamma) \nabla\left(\nabla^{2} \rho\right)+\kappa^{\prime}(\gamma) \boldsymbol{g}\right\}
$$

The components of the two-dimensional vector $\boldsymbol{g}$ in the last equation are

$$
g_{x}=\frac{\partial \mathcal{K}}{\partial x} \frac{\partial^{2} \rho}{\partial y^{2}}-\frac{\partial \mathcal{K}}{\partial y} \frac{\partial^{2} \rho}{\partial x \partial y}
$$

and

$$
g_{y}=\frac{\partial \mathcal{K}}{\partial y} \frac{\partial^{2} \rho}{\partial x^{2}}-\frac{\partial \mathcal{K}}{\partial x} \frac{\partial^{2} \rho}{\partial x \partial y},
$$

where $\mathcal{K}=\operatorname{det}\left(\rho_{i j}\right)$. This is just the equation of continuity with a constitutive equation for the mass current

$$
\boldsymbol{J}=D(\gamma)\left(1-B|\nabla \rho|^{2}\right) \nabla \rho+\kappa(\gamma) \nabla\left(\nabla^{2} \rho\right)+\kappa^{\prime}(\gamma) \boldsymbol{g}
$$

However $\boldsymbol{g}$ given by (4) and (5) is not unique and $\boldsymbol{g}$ is chosen in the above form to avoid calculating fifth derivatives numerically later in the simulation. Alternative forms of $\boldsymbol{g}$ can give rise to the same equation for $\rho$ on substituting into (3). These forms of $\boldsymbol{g}$ differ from the form given above by an additional gauge term which is the curl of an arbitrary vector. One example of these alternative forms is found by interchanging $\mathcal{K}$ and $\rho$ in (44) and (15).

We now give a physical interpretation of the terms in the free energy functional. The first term and the second term give the diffusion term proportional to $\nabla \rho$ with limiting factor $\left(1-B|\nabla \rho|^{2}\right)$ in (3). The diffusion term must have the opposite sign to the usual (if $|\nabla \rho|$ is small and the limiting factor is positive) in order to mimic the short-range attractive force between particles. Thus the coefficient $D$ must be positive so that grains diffuse toward each other to form clusters. The limiting factor $\left(1-B|\nabla \rho|^{2}\right)$ can be understood as the result of anisotropy in the system due to gravity and ensures that the slope of the heaps cannot exceed the critical slope. Because of the opposite sign of the diffusion term the equation can be understood as a negative time diffusion equation.

The last two terms in $\mathcal{F}$ come from the squares of the two rotational invariants of the Hessian matrix $\rho_{i j}$. These invariants are the trace and the determinant of $\rho_{i j}$. They are lowest order approximations to the average and Gaussian curvatures of the surface and we now refer to them as such. We can understand these curvature terms intuitively thus. As the system is tapped protrusions are eroded so that the sides of the heaps becomes as smooth as possible i. e. the curvatures of the surface become as small as possible. Equation (2) ensures the 
system evolves to minimise the curvatures of the surface. There are no linear terms in $\mathcal{F}$ proportional to the trace and determinant because as we can show, with periodic (or free) boundaries, these give no contribution to the equation of motion of $\rho$.

\section{Calculations}

We now solve (3) with (44) and (5) numerically by discretising the spatial variables on an $N \times N$ square lattice using a simple mid-point algorithm to deal with the time variable. We can use either periodic boundary conditions or hard boundary conditions. However we are only interested here in the pattern formation process, not the interaction of the system with the container wall so here we use only periodic boundary conditions. Various initial conditions are possible but here we start off with a level granular layer with random height fluctuations of amplitude $10^{-5}$ superimposed. This amplitude is small compared to the final average height of the heaps which is determined by the value of the parameter $B$ and the length of time for which the calculation is run.

Stability problems arise if $\kappa$ is negative. This can be easily seen if we take the Fourier transform of the linearised equation which is

$$
\frac{\partial \rho_{k}}{\partial t}=\left(D k^{2}-\kappa k^{4}\right) \rho_{k}
$$

If $\kappa$ is positive small fluctuations of long-wave-length in the initial state are unstable to growth until limited by the non-linear term proportional to $B$ in $\boldsymbol{J}$. This is in accordance with observation. If $\kappa$ is negative small fluctuations of long-wave-lengths in the initial state grow and are not limited by the non-linear term because the term proportional to $\kappa$ dominates. Negative values of $\kappa$ and $\kappa^{\prime}$ are thus quite unphysical because they imply that protrusions are not eroded away but grow indefinitely as they accrete grains in a situation where there is no real attractive force between grains.

As time increases instabilities develop from the density fluctuations and grow into heaps. While the heaps grow grains from the surrounding areas are depleted. This creates discontinuities in the layer and introduces extra complications when calculating derivatives of the density field. To avoid this we assume in the simulation that the surface of the layer never touches the base of the container. The assumption enables us to avoid these problems but it has a draw-back since the heaps will continue growing until the whole system unrealistically becomes a single big heap. In reality the surface of the layer eventually touches the base of the container and the heaps become very nearly stable and cease to grow.

In the following we take a box size $L=1$ divided into $10^{4}$ cells so that $N=100$. We take the following values for the parameters: $D=1, B=10^{3}$, $\kappa=10^{-4}$, and we vary $\kappa^{\prime}$ so that we can see the balance between the terms affecting the occurrence of heaps and ridges. We use periodic boundary conditions throughout.

Figure 2 shows late-time plots of the surface for values of the parameters where the Gaussian curvature term is unimportant $\left(\kappa^{\prime}=10^{-6}\right)$. The appearance 

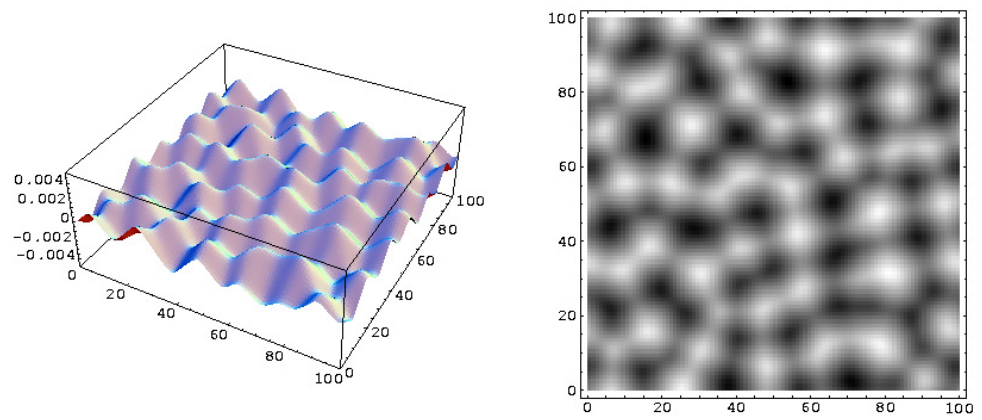

Fig. 2. A three-dimensional plot and a grey-scale contour plot of the surface produced by our differential equation after a long time. The parameters used to produce the plot favour the production of heaps i. e. $\kappa^{\prime} \ll \kappa$.
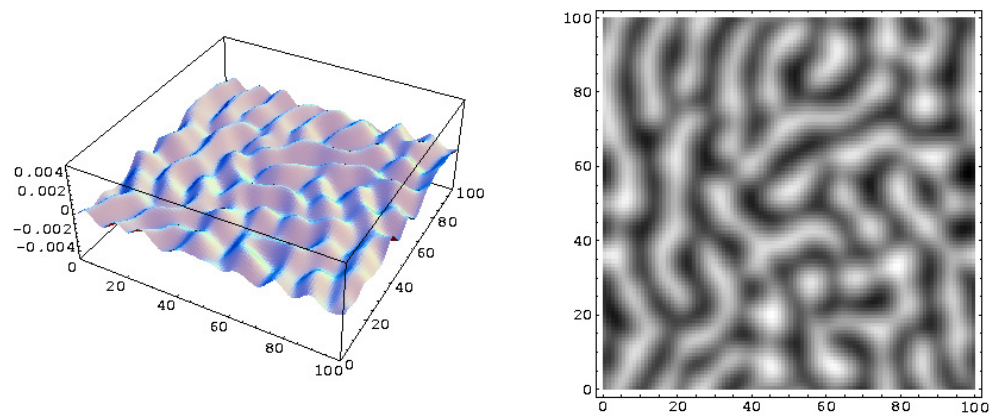

Fig. 3. A three-dimensional plot and a grey-scale contour plot of the surface produced by our differential equation after a long time. The parameters used to produce the plot favour the production of ridges i. e. when $\kappa^{\prime}$ is comparable to $\kappa$ (in this case $\kappa^{\prime}=\kappa$ ).

of the heaps is similar to those found experimentally except that our calculations display structures that are more homogeneous which is to be expected for a surface with no edges and periodic boundary conditions. Figure 3 shows late-time plots of the surface for values of the parameters where the Gaussian curvature term is important $\left(\kappa^{\prime}=10^{-4}\right)$. The resulting structure is a pattern of labyrinthine ridges. The pattern is similar to the patterns that have been found in the early stage of the experiments performed (c.f. Figure 1 ). An interesting feature of these last figures from a theoretical point of view is that they show long-range correlations which arise from a purely local theory. The term discouraging saddles leads to long-range correlations along the ridges.

The differences in the morphologies of the results can be understood by looking at the surface free energy in (1). Equation (3) is derived by minimising the 


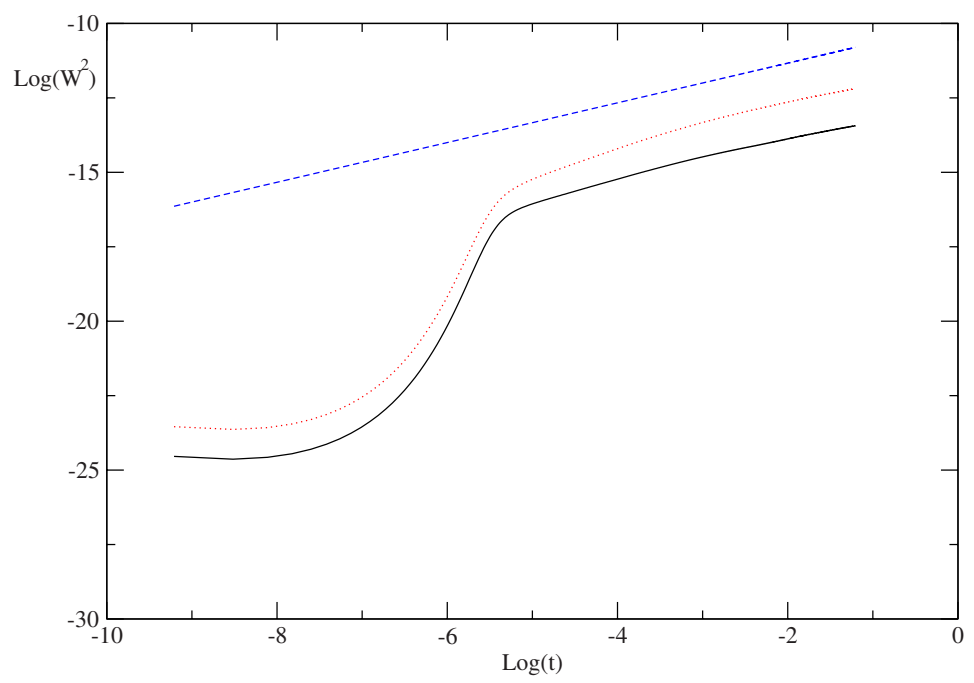

Fig. 4. Log-log plot for $\mathcal{W}^{2}$ versus $t$. The solid line corresponds to the parameter $\kappa^{\prime}=10^{-6}$, the dotted line corresponds to $\kappa^{\prime}=10^{-4}$ and they are offset vertically by unity for clarity. A reference line (dashed) with slope $2 / 3$ is plotted to aid comparison with the late-time behaviour for both small and large $\kappa^{\prime}$ curves.

functional $\mathcal{F}[\rho]$, and therefore the dynamic of the system is towards minimal surface curvature. For small values of $\kappa^{\prime}$, the trace term is important and $\left[\operatorname{tr}\left(\rho_{i j}\right)\right]^{2}$ is small at locations where two local perpendicular curvatures curve in opposite directions $\partial^{2} \rho / \partial y^{2}=-\partial^{2} \rho / \partial x^{2}$, i.e. at saddle points or where the surface is smooth. Thus the equation produces a profusion of saddle points. Thus the fact that two heaps (or two valleys) join at a saddle point suggests that minimising the trace term encourages the formation of as many heaps as possible. On the other hand, for slightly larger values of $\kappa^{\prime}$, the determinant term encourages the formation of ridges. This can be seen from a similar argument to that used for the trace term. We can always choose a local reference frame $\left(x^{\prime}, y^{\prime}\right)$ such that $\operatorname{det}\left(\rho_{i j}\right)=\partial^{2} \rho / \partial x^{\prime 2} \partial^{2} \rho / \partial y^{\prime 2}$. Minimising the determinant term in (1) requires $\partial^{2} \rho / \partial x^{\prime 2} \partial^{2} \rho / \partial y^{\prime 2}=0$ which means the surface is either flat in one direction but is curved in the perpendicular direction (which is true for ridges); or the surface is flat in both directions (but unstable due to the diffusion term).

Figure 4 shows a log-log plot of the roughness $\mathcal{W}^{2}$ of the pattern for both small $\left(\kappa^{\prime}=10^{-6}\right)$ and large $\left(\kappa^{\prime}=10^{-4}\right)$ values of $\kappa^{\prime}$. Here $\mathcal{W}^{2}$ is defined as

$$
\mathcal{W}^{2}=\left\langle(\rho(\boldsymbol{x}, t)-\bar{\rho})^{2}\right\rangle,
$$

where the average is over all lattice sites. $\mathcal{W}^{2}$ measures the mean square deviation of the height of the surface from its mean and is zero for a level surface and grows as the heaplets grow. $\mathcal{W}^{2}$ is proportional to the square of the characteristic length scale of the pattern in the horizontal direction because the slope of the surface is almost everywhere equal to the critical slope $1 / \sqrt{B}$. As can be seen from 
Figure 4, the dynamics of (3) can be divided into at least 3 regimes. For $t<10^{-7}$, the system tends to become smoother because slopes greater than the critical slope decrease. For $10^{-7}<t<10^{-5}$ there is a rapid formation of heaps due to the growth of fluctuations. For $t>10^{-5}$ the heaps have reached their critical slope and then grow coarser but with the fixed critical slope almost everywhere. In the late-time regime, $\mathcal{W}^{2} \sim t^{2 / 3}$. This scaling law is consistent with Siegert and Plishke's model [11] of molecular-beam epitaxy deposition. Despite an extra deposition term and the absence of a Gaussian term in their model, they have observed the same scaling law for the characteristic size of the mound formed in the deposition process.

\section{Conclusion}

We have studied a phenomenological model of a tapped granular layer. The model is derived by minimising a surface free energy consisting of four terms: two terms which give rise to the slope-limited negative-time diffusion, the average curvature term, and the Gaussian curvature term. The negative-time diffusion terms mimic the clustering effects described also by an effective surface tension. The curvature terms are needed because the system must evolve to a configuration of less protrusion. They also control the pattern formed: a striped pattern if the Gaussian curvature term is important; discrete heaplets if the average curvature term is dominant. In both cases the pattern coarsens with the number of taps and the coarsening of the width of the pattern is described by $\mathcal{W} \sim t^{1 / 3}$.

\section{References}

1. Melo, F., Umbanhowar, P.B., Swinney, H.L.: Hexagons, kinks, and disorder in oscillated granular layer. Phys. Rev. Lett. 75 (1995) 3838

2. Umbanhowar, P.B., Melo, F., Swinney, H.L.: Localised excitation in a vertically vibrated granular layer. Nature 382 (1996) 793

3. Knight, J.B., Fandrich, C.G., Lau, C.N., Jaegar, H.M., Nagel, S.R.: Density relaxation in a vibrated granular material. Phys. Rev. E 51 (1995) 3957

4. Nowak, E.R., Knight, J.B., Ben-Naim, E., Jaeger, H.J., Nagel, S.R.: Density fluctuations in vibrated granular materials. Phys. Rev. E 57 (1998) 1971

5. Makse, H.A., Havlin, S., King, P.R., Stanley, H.E.: Spontaneos stratification in granular mixtures. Nature 386 (1997) 379

6. Ristow, G.H., Riguidel, F.X., Bideau, D.: Different characteristics of the motion of a single particle on a bumpy inclined line. J. Phys. II France 4 (1994) 1161

7. Duran, J.: Rayleigh-Taylor instabilities in thin films of tapped powder. Phys. Rev. Lett. 87 (2001) 254301

8. Duran, J.: Ripples in tapped or blown powder. Phys. Rev. Lett. 84 (2000) 5126

9. Goh, Y.K., Jacobs, R.L.: Coarsening dynamics of granular heaplets in tapped granular layers. New J. Phys. 4 (2002) 81

10. Gunton, J.D., Droz, M.: Introduction to the theory of metastable and unstable states. Volume 183 of Lecture notes in physics. Springer-Verlag, Berlin (1983)

11. Siegert, M., Plischke, M.: Formation of pyramids and mounds in molecular beam epitaxy. Phys. Rev. E 53 (1996) 307 